TRANSACTIONS OF THE

AMERICAN MATHEMATICAL SOCIETY

Volume 359, Number 2, February 2007, Pages 687-700

S 0002-9947(06)03890-6

Article electronically published on August 16, 2006

\title{
HOMOTOPICAL COMPLEXITY AND GOOD SPACES
}

\author{
M. INTERMONT AND J. STROM
}

\begin{abstract}
This paper is an exploration of two ideas in the study of closed classes: the $A$-complexity of a space $X$ and the notion of good spaces (spaces $A$ for which $\mathcal{C}(A)=\overline{\mathcal{C}(A)}$ ). A variety of formulae for the computation of complexity are given, along with some calculations. Good spaces are characterized in terms of the functors $C W_{A}$ and $P_{A}$. The main result is a countable upper bound for $\Sigma A$-complexity when $A$ is a good space.
\end{abstract}

\section{INTRODUCTION}

Our purpose in this paper is to explore and bring together two distinct strands in the study of closed classes, namely the complexity of a space $X$ with respect to another space $A$ and the notion of good spaces.

A closed class is a class $\mathcal{C}$ of pointed topological spaces which is closed under weak equivalences and pointed homotopy colimits. The main examples of closed classes are those of the form $\mathcal{C}(A)$, the smallest closed class containing the space $A$; in fact, every known example of a closed class has this form. The spaces $X \in$ $\mathcal{C}(A)$ are called $A$-cellular spaces; such spaces can be 'built' from $A$ by repeatedly performing homotopy colimits, and this relation is often denoted $A \ll X$. For an arbitrary space $X$ and a fixed $A$, while $X$ may not be in $\mathcal{C}(A)$, there is a closest $A$-cellular approximation to $X$, which is denoted $C W_{A}(X)$.

The $A$-complexity of $X$, denoted $\kappa_{A}(X)$, is the minimum (ordinal) number of homotopy colimit operations needed to construct $C W_{A}(X)$ starting with wedges of copies of $A\left[\mathrm{CDI}\right.$. Thus $\kappa_{A}(X)$ measures how difficult it is to build $X$ from $A$. In principle, this invariant can take on arbitrarily large ordinal number values. However, a theorem of Stover $\left[\mathrm{S}\right.$ ] implies that when $A=S^{1}$, the $S^{1}$-complexity of any space $X$ is at most 1 . More generally, the argument can be extended to show that $\kappa_{S^{n}}(X) \leq 2$ for every space $X$ and every $n \geq 0$ [CDI. It is shown in the same paper that there are spaces $X$ for which $\kappa_{M(\mathbb{Z} / p, n)}(X)=\omega$, the first infinite ordinal number.

If $\mathcal{C}$ is a closed class, then we may add just enough spaces to it in order to ensure that it is also closed under extensions by fibrations; we write $\overline{\mathcal{C}}$ to denote the resulting strongly closed class. If it happens that $\mathcal{C}(A)=\overline{\mathcal{C}(A)}$, then we say that $A$ is a good space. For example, $\mathcal{C}\left(S^{n}\right)$ is easily seen to consist of all $(n-1)$ connected spaces, which is evidently closed under extensions by fibrations; hence $S^{n}$ is a good space. Furthermore, a recent result of Chachólski, Parent and Stanley [CPS] shows that for every space $A$, there is a space $B$ such that $\overline{\mathcal{C}(A)}=\mathcal{C}(B)$;

Received by the editors June 10, 2004 and, in revised form, November 23, 2004.

2000 Mathematics Subject Classification. Primary 55Q05.

Key words and phrases. Closed class, complexity, homotopy colimit.

(C)2006 American Mathematical Society 687 
thus there are many good spaces. On the other hand it is known that the Moore space $M(\mathbb{Z} / p, n)$ is not a good space. Good spaces are not very well understood: for example, it is not known whether the wedge of two good spaces is also good.

There are three main sections: $\S 2$ is concerned with $A$-complexity; $\S 3$ is devoted to the study of good spaces; and in $\S 4$ we bring these two themes together and prove our main theorem on complexity with respect to good spaces.

In $\S 2$, we develop formulae which facilitate the calculation, or at least the estimation, of $A$-complexity. We offer two results here as examples.

Theorem 16. If $A \ll X$, then

(a) $\Omega A \ll \Omega X$ (due to Chachólski [C2]), and

(b) $\kappa_{\Omega A}(\Omega X) \leq 1+\kappa_{A}(X)$.

Theorem 18. If $X \sim \Omega Y$ for some space $Y$ and $\Sigma A \ll \Sigma X$, then

(a) $A \ll X$ (due to Chachólski C3]), and

(b) $\kappa_{\Sigma A}(\Sigma X) \leq \kappa_{A}(X) \leq \kappa_{\Sigma A}(\Sigma X)+2$.

We use our formulae to make some explicit computations, including, for example, that $\kappa_{K(G, n)}(K(G, n+m)) \leq 2 m$ for Eilenberg-Mac Lane spaces.

Then in $\S 3$ we turn to the study of good spaces. There are three main results in this section. First, we show that if $A$ is a good space, then so is $\Sigma A$. Next we characterize good spaces in terms of the $A$-cellularization functor $C W_{A}$ and the $A$-nullification functor $P_{A}$.

Theorem 23. A space $A$ is good if and only if the natural sequence

$$
C W_{A}(X) \rightarrow X \rightarrow P_{A}(X)
$$

is a fibration sequence for every space $X$.

Finally, we use these results to give an efficient construction of the functor $P_{A}$ under the assumption that $A$ is a good space.

In the fourth and final section we bring together results from $\S \S 2$ and 3 in order to prove our main result: a countable upper bound for the complexity of spaces with respect to the suspension of a good space.

Theorem 16. If $A$ is a good space, then

$$
\kappa_{\Sigma A}(X) \leq \omega+1 \quad \text { for every space } X .
$$

As a corollary, we show that if $A$ is good and $A \ll \Omega Y$, then $\kappa_{A}(\Omega Y) \leq \omega+1$.

\section{Preliminaries}

In this section we establish our notation and conventions and recall the basic definitions which we use throughout the paper.

1.1. Conventions. Unless otherwise stated, all spaces and maps in this paper are pointed; all basepoints are denoted by $*$. We use $\sim$ to denote weak equivalence. The notation $\operatorname{map}_{*}(X, Y)$ indicates the space of pointed maps from $X$ to $Y$.

We write $\mathbf{S p a c e s}_{*}$ to denote the category of pointed spaces and pointed continuous maps; Spaces is then the category of unpointed spaces and unpointed continuous maps. We will need to refer to both pointed and unpointed homotopy colimits: we write hocolim ${ }_{*}$ for the pointed homotopy colimit and hocolim for the unpointed homotopy colimit. We refer to Bousfield and Kan [B-K] for the definition and basic properties of homotopy colimits. 
Our formulae involve ordinal number arithmetic, for which we refer to $\mathrm{Fr}$, Ch. III, $\S 10]$. Addition of ordinal numbers is not commutative: for example $1+\omega=\omega \neq$ $\omega+1$, where $\omega$ is the first infinite ordinal [Fr, p. 142]. Each ordinal number $\alpha$ corresponds to a small category whose objects are the ordinals $\beta<\alpha$, and whose morphisms $\beta \rightarrow \delta$ correspond to inequalites $\beta \leq \delta$. We will use the same letter to refer to the ordinal number and the category; thus a functor $\Phi: \omega \rightarrow \mathbf{S p a c e s}_{*}$ is a pointed telescope diagram.

\subsection{Good spaces.}

Definition 1. A class $\mathcal{C}$ of pointed spaces is called a closed class if it is closed under weak equivalences and pointed homotopy colimits; we say that $\mathcal{C}$ is strongly closed if, in addition, it is closed under extensions by fibrations; that is, if $F \rightarrow$ $E \rightarrow B$ is a fibration sequence with $F, B \in \mathcal{C}$, then $E \in \mathcal{C}$.

The main examples of closed classes are the classes $\mathcal{C}(A)$, the smallest closed class containing the space $A$. The spaces in $\mathcal{C}(A)$ are called $A$-cellular. The relation $B \in \mathcal{C}(A)$ is often denoted $A \ll B$. For any closed class $\mathcal{C}$, we denote the smallest strongly closed class containing $\mathcal{C}$ by $\overline{\mathcal{C}}$.

Definition 2. A space $A$ is good if $\mathcal{C}(A)=\overline{\mathcal{C}(A)}$.

There is an augmented functor $C W_{A}: \mathbf{S p a c e s}_{*} \rightarrow \mathbf{S p a c e s}_{*}$ which assigns to a space $X$ its 'best approximation' by an $A$-cellular space [Fa2, Ch. 2]. In particular, the map $C W_{A}(X) \rightarrow X$ induces a weak equivalence $\operatorname{map}_{*}\left(A, C W_{A}(X)\right) \rightarrow$ $\operatorname{map}_{*}(A, X)$; any map which induces a weak equivalence on $\operatorname{map}_{*}(A,-)$ is called an $A$-equivalence. Related to $C W_{A}$ there is the nullification functor $P_{A}$ which assigns to $X$ its 'best approximation' by a space with $\operatorname{map}_{*}\left(A, P_{A}(X)\right) \sim *[\mathrm{~B}$.

1.3. A-complexity. The $A$-complexity of a space was defined and studied in the paper [CDI]. The definition is made in terms of certain full subcategories $\mathcal{C}_{\alpha}^{A}$ of the category Spaces $_{*}$. Since these subcategories are full, it suffices to specify their objects, and we begin by setting

$$
\operatorname{Obj}_{0}^{A}=\{X \mid X \sim \text { a retract of a wedge of copies of } A\} .
$$

Having defined $\mathcal{C}_{\beta}$ for all ordinal numbers $\beta<\alpha$, we set

$$
\operatorname{Obj}_{\alpha}^{A}=\left\{X \mid X \sim \text { a retract of hocolim }{ }_{*} \Phi \text { where } \Phi: \mathcal{D} \rightarrow \mathcal{C}_{<\alpha}^{A}\right\},
$$

where $C_{<\alpha}=\bigcup_{\beta<\alpha} C_{\beta}^{A}$, D runs over all small categories and $\Phi$ runs over all functors.

Definition 3. The $A$-complexity of $X$ is the ordinal number

$$
\kappa_{A}(X)=\min \left\{\alpha \mid C W_{A}(X) \in \mathcal{C}_{\alpha}^{A}\right\} .
$$

It follows from the construction of the functor $C W_{A}[\mathrm{Fa} 2, \S 2 . \mathrm{B}]$ that $\mathcal{C}(A)=\mathcal{C}_{\alpha}^{A}$ for sufficiently large $\alpha$ [CDI, Prop. 1.5]; hence every space $X$ has a well-defined $A$-complexity.

We point out that we have begun using what will be a consistent abuse of notation: writing $X \in \mathcal{C}_{\alpha}^{A}$ to mean $X \in \operatorname{Obj}_{\mathcal{\alpha}}^{A}$. 


\section{Formulas FOR COMPLEXITY}

The paper [CDI] established a theoretical framework for complexity, but did not seek to provide methods for its calculation. Our purpose in this section is to develop formulae that that will allow for the computation, or at least the estimation, of complexity.

We begin with two simple but crucial lemmas. The first concerns complexity with respect to $S^{0}$.

Lemma 4. For any space $X, S^{0} \ll X$ and $\kappa_{S^{0}}(X) \leq 1$.

Proof. First replace $X$ with a weakly equivalent simplicial complex; such a replacement necessarily has the same $A$-complexity. Let $\mathbf{X}$ denote the simplex category of $X$; then $X$ is weakly equivalent to the unpointed homotopy colimit of the functor $\Phi: \mathbf{X} \rightarrow$ Spaces defined by $\Phi(\sigma)=*$ for each simplex $\sigma$ of $X$. If we attach disjoint basepoints throughout, we obtain the space $X_{+}$( $X$ with a disjoint basepoint) as a pointed homotopy colimit of $\Theta: \mathbf{X} \rightarrow$ Spaces $_{*}$ with $\Theta(\sigma)=S^{0}$ for each $\sigma$ in $X$. If $x \in X$ is the basepoint (which we take to be a vertex), then $X$ is the cofiber of natural map $\{x\}_{+} \rightarrow X_{+}$; thus we may identify the external basepoint of $X_{+}$with the given basepoint of $X$ by enlarging the diagram slightly.

Our second lemma is a simple transitivity formula.

Lemma 5. If $A \ll X \ll Y$, then $\kappa_{A}(Y) \leq \kappa_{A}(X)+\kappa_{X}(Y)$.

Proof. Write $\kappa_{A}(X)=\alpha$ and $\kappa_{X}(Y)=\beta$. Then, since $X$ is $A$-cellular, $X \in \mathcal{C}_{\alpha}^{A}$. It follows that $\mathcal{C}_{0}^{X} \subseteq \mathcal{C}_{\alpha}^{A}$, and hence that $Y \in \mathcal{C}_{\beta}^{X} \subseteq \mathcal{C}_{\alpha+\beta}^{A}$.

2.1. Wedges, smashes and products. We begin by giving some formulae for the behavior of complexity with respect to some basic operations of homotopy theory.

Wedges are easily dispensed with.

Proposition 6. For any spaces $X_{i} \in \mathcal{C}(A), \kappa_{A}\left(\bigvee_{i} X_{i}\right)=\operatorname{supremum}\left\{\kappa_{A}\left(X_{i}\right)\right\}$.

Proof. Since $\mathcal{C}_{0}^{A}$ is closed under wedges, so is $\mathcal{C}_{\alpha}^{A}$ for all $\alpha$.

We now consider suspensions and, more generally, smash products.

Proposition 7. If $A \ll X$, then $A \ll \Sigma A \ll \Sigma X$, and

(a) $\kappa_{\Sigma A}(\Sigma X) \leq \kappa_{A}(X)$, and

(b) if $\kappa_{A}(X) \geq 1$, then $\kappa_{A}(\Sigma X) \leq \kappa_{A}(X)$.

Proof. Part (a) follows instantly from the fact that suspension commutes with homotopy colimits. For part (b), we simply observe that $\mathcal{C}_{\alpha}^{A}$ is closed under suspension for $\alpha \geq 1$.

When $\kappa_{A}(X)=0$, we cannot conclude that $\kappa_{A}(\Sigma X)=0$. The best possible estimate, $\kappa_{A}(\Sigma X) \leq 1$, follows from a much more general result about smash products.

Proposition 8. If $A \ll Y$, then for any space $X$,

(a) $A \ll X \wedge Y$, and

(b) $\kappa_{A}(X \wedge Y) \leq \kappa_{A}(Y)+1$. 
Proof. Part (a) is well known [Fa2, Thm. 2.D.8]. For (b), write $\kappa_{A}(Y)=\alpha$, so $\mathcal{C}_{0}^{Y} \subseteq C_{\alpha}^{A}$, and hence $\mathcal{C}_{\beta}^{Y} \subseteq \mathcal{C}_{\alpha+\beta}^{A}$ for all $\beta$. Since $X \in \mathcal{C}_{1}^{S^{0}}$ by Lemma 4 it follows immediately that $X \wedge Y \in \mathcal{C}_{1}^{S^{0} \wedge Y} \subseteq \mathcal{C}_{\alpha+1}^{A}$, which means that $\kappa_{A}(X \wedge Y) \leq \alpha+1$.

We have the following improvement when both spaces are $A$-cellular.

Corollary 9. If $A \ll X, Y$, then

(a) $A \ll X \wedge Y$, and

(b) $\kappa_{A}(X \wedge Y) \leq \min \left\{\kappa_{A}(X), \kappa_{A}(Y)\right\}+1$.

The fact that closed classes are closed under cartesian products is one of their most important features [Fa2, 2.D.15]. We finish this subsection with estimates for the complexity of a product.

Proposition 10. Let $A \ll X, A \ll Y$ and let $M=\max \left\{\kappa_{A}(X), \kappa_{A}(Y)\right\}$. Then

(a) $A \ll X \times Y$,

(b) $M \leq \kappa_{A}(X \times Y) \leq M+\omega$, and

(c) if $A \sim \Sigma B$, then $M \leq \kappa_{A}(X \times Y) \leq 2+M$.

Proof. The cellular inequality (a) is well known. The lower bound $M \leq \kappa_{A}(X \times Y)$ is valid because $X$ and $Y$ are both retracts of $X \times Y$.

For (b) we recall the proof of the cellular inequality given in [Fa2, Thm. 2.D.16]. Replace $X$ and $Y$ with weakly equivalent CW complexes if necessary. Set $P(n)=$ $\{*\} \times Y \cup X \times Y_{n}$ and observe that $P(n+1)$ is the (homotopy) pushout of a diagram

$$
X \rtimes D^{n+1} \leftarrow X \rtimes S^{n} \rightarrow P(n) .
$$

Now $P(0)=X \vee Y \in \mathcal{C}_{M}^{A}, X \rtimes D^{n+1} \sim X \in \mathcal{C}_{M}^{A}$ and $X \rtimes S^{n} \sim X \wedge S_{+}^{n} \in \mathcal{C}_{M+1}^{A}$ by Proposition 8 (b). It follows by induction that $P(n) \in \mathcal{C}_{M+n}^{A}$ for all $n$. The inclusions $P(n) \hookrightarrow P(n+1)$ constitute a telescope $\Psi: \omega \rightarrow$ Spaces $_{*}$ whose homotopy colimit is $X \times Y$. Thus $\kappa_{A}(X \times Y) \leq M+\omega$, as desired.

The proof of (c) is an instance of a general approach to products. Let $\mu$ be the supremum of $\kappa_{A}(P \times Q)$ for $P, Q \in \mathcal{C}_{0}^{A}$. We argue by induction that if $X, Y \in \mathcal{C}_{\alpha}^{A}$, then $\alpha \leq \kappa_{A}(X \times Y) \leq \mu+\alpha$. The case $\kappa_{A}(X)=\kappa_{A}(Y)=0$ is taken care of by the definition of $\mu$. In the inductive case, we have

$$
X \sim \operatorname{hocolim}_{*} \Phi_{X} \quad \text { and } \quad Y \sim \operatorname{hocolim}_{*} \Phi_{Y},
$$

where $\Phi_{X}: \mathcal{I} \rightarrow$ Spaces $_{*}$ and $\Phi_{Y}: \mathcal{J} \rightarrow$ Spaces $_{*}$ with $\Phi_{X}(i), \Phi_{Y}(j) \in \mathcal{C}_{<\alpha}^{A}$ for all $i \in \mathcal{I}$ and $j \in \mathcal{J}$. (Actually, $X$ and $Y$ could be retracts of such spaces, but then $X \times Y$ is a retract of a product of such spaces, so we suffer no loss of generality.) Define $\Theta: \mathcal{I} \times \mathcal{J} \rightarrow \mathbf{S p a c e s}_{*}$ by the formula $\Theta(i, j)=\Phi_{X}(i) \times \Phi_{Y}(j)$. For each $i \in \mathcal{I}$ and $j \in \mathcal{J}$ there is a $\beta<\alpha$ with $\Phi_{X}(i), \Phi_{Y}(j) \in \mathcal{C}_{\beta}^{A}$. It follows from the inductive hypothesis that $\Theta(i, j) \in \mathcal{C}_{\mu+\beta}^{A} \subseteq \mathcal{C}_{<\mu+\alpha}^{A}$ because $\mu+\beta<\mu+\alpha$ [Fr. p. 208]. Hence $X \times Y \sim \operatorname{hocolim}_{*} \Theta \in \mathcal{C}_{\mu+\alpha}^{A}$.

In view of the previous paragraph, the proof of (c) will be complete if we simply verify the upper bound in the initial case, $\alpha=0$. Here $X$ and $Y$ are both (retracts of) wedges of suspensions of $B$. Thus $[\mathrm{A}$ the product can be obtained by the cofiber sequence

$$
\Sigma^{-1} X * \Sigma^{-1} Y \rightarrow X \vee Y \rightarrow X \times Y \text {. }
$$

Since $\Sigma^{-1} X * \Sigma^{-1} Y \cong X \wedge\left(\Sigma^{-1} Y\right)$, we have $\kappa_{A}\left(\Sigma^{-1} X * \Sigma^{-1} Y\right) \leq \kappa_{A}(X)+1=1$ by Proposition 8 (b). Since $\kappa_{A}(X \vee Y)=0$, it follows that $\kappa_{A}(X \times Y) \leq 2$. 
2.2. E. Dror Farjoun's theorem and some of its consequences. Many of the most striking cellular inequalities are proved using the following theorem of E. Dror Farjoun [Fa2, Thm.9.A.8] (see also [Fa1, Thm. 8.1]). The only novelty in our statement of the theorem is the inclusion of a complexity estimate.

Theorem 11. Let $\mathcal{K}$ be a small category, and let

$$
\mathbf{E}: \mathcal{K} \rightarrow \text { Spaces } \quad \text { and } \quad \mathbf{B}: \mathcal{K} \rightarrow \text { Spaces }
$$

be two diagrams whose (unpointed) homotopy colimits are the spaces $E$ and $B$, respectively. Let $\mathbf{f}: \mathbf{E} \rightarrow \mathbf{B}$ be a natural transformation which induces the map $f: E \rightarrow B$. For each $k \in \mathcal{K}$, let $W_{k}$ denote the homotopy fiber of $\mathbf{f}_{k}$, and write $W=\bigvee_{\kappa \in \mathcal{K}} W_{k}$. If $F$ is the homotopy fiber of $f$, then

(a) $W \ll F$, and

(b) $\kappa_{W}(F) \leq 1$.

Proof. As mentioned above, part (a) is already known [Fa1]. To prove part (b), we recall that (a) is proved by constructing an explicit diagram $\Phi: \mathcal{L} \rightarrow \mathbf{S p a c e s}_{*}$ whose homotopy colimit is weakly equivalent to $F$ and whose pointwise values are the spaces $W_{k}$. Since each $W_{k} \in \mathcal{C}_{0}^{W}$, we have $F \in \mathcal{C}_{1}^{W}$.

This is the general outline of many of the proofs in this section: simply examine the proof of a known cellular inequality and count the number of homotopy colimits that are used.

The following is a useful instant corollary of Theorem 11

Corollary 12. Let $A \rightarrow B \rightarrow C$ be a cofiber sequence and let $F$ be the homotopy fiber of the map $B \rightarrow C$. Then

(a) $A \ll F$, and

(b) $\kappa_{A}(F) \leq 1$.

Next we consider relations between the fiber and the cofiber of a map; the cellular inequality is from E. Dror Farjoun's paper [Fa1].

Theorem 13. Let $f: X \rightarrow Y$ be a map with $Y$ path connected. Denote the homotopy fiber and cofiber of $f$ by $F$ and $C$, respectively. Then

(a) $\Sigma F \ll C$, and

(b) $\kappa_{\Sigma F}(C) \leq 1$.

Proof. The proof the cellular inequality given in [C2, Prop. 10.5] uses Theorem 11] once.

Theorem 14. For any path connected space $X$,

(a) $X \ll \Omega \Sigma X$, and $\kappa_{X}(\Omega \Sigma X) \leq 1$, and

(b) $\Sigma \Omega X \ll X$, and $\kappa_{\Sigma \Omega X}(X) \leq 1$.

Proof. The cellular inequality of part (a) appeared in [Fa1]; the proof given in C2, Prop. 10.7] uses Theorem 11 to present $\Omega \Sigma X$ as a pointed homotopy colimit of a diagram whose pointwise values are the spaces $X$ and $*$. Part (b) follows by applying Theorem 13 to the map $* \rightarrow X$. 
2.3. A replacement for $\mathcal{C}_{0}^{A}$. In order to obtain formulae which control the behavior of loop spaces, we use a modified definition of complexity. Rather than beginning with $\mathcal{C}_{0}^{A}$, we begin with the full subcategory whose objects are

$$
\operatorname{Obj}\left(\widetilde{\mathcal{C}}_{0}^{A}\right)=\{A, *\} .
$$

We denote the resulting notion of complexity by $\widetilde{\kappa}_{A}$. It is not hard to verify that the results of $\S \S 2.2$ and 2.3 are valid for the invariant $\widetilde{\kappa}_{A}$ (with the exception of Proposition 6 in the case $\widetilde{\kappa}_{A}\left(X_{i}\right)=0$ for all $\left.i\right)$.

Proposition 15. For any space $X, \kappa_{A}(X) \leq \widetilde{\kappa}_{A}(X) \leq 1+\kappa_{A}(X)$.

Proof. This follows from the obvious containments $\mathcal{C}_{0}^{A} \subseteq \widetilde{\mathcal{C}}_{1}^{A} \subseteq \mathcal{C}_{1}^{A}$.

In C2, Chachólski proved the surprising result that cellular inequalities are preserved by the loop space operation. We use our modified invariant to estimate the complexity $\kappa_{\Omega A}(\Omega X)$.

Theorem 16. If $A$ is connected and $A \ll X$, then

(a) $\Omega A \ll \Omega X$, and

(b) $\kappa_{\Omega A}(\Omega X) \leq 1+\kappa_{A}(X)$.

Proof. We observe first that, in view of Proposition 15, it suffices to prove that $\widetilde{\kappa}_{\Omega A}(\Omega X) \leq \widetilde{\kappa}_{A}(X)$.

If $\widetilde{\kappa}_{A}(X)=0$, then $X \sim A$ or $X \sim *$; in either case $\widetilde{\kappa}_{\Omega A}(\Omega X)=0$. Let us assume inductively that $\widetilde{\kappa}_{\Omega A}(\Omega X) \leq \widetilde{\kappa}_{A}(X)$ for all $X \in \widetilde{\mathcal{C}}_{<\alpha}^{A}$.

The proof of statement (a) given in [C2, Cor. 10.4] uses Theorem 11$]$ to show that if $X \sim \operatorname{hocolim}_{*} \Phi$, where $\Phi: \mathcal{K} \rightarrow$ Spaces $_{*}$, then $\Omega X \sim \operatorname{hocolim}_{*} \Theta$, where $\Theta: \mathcal{L} \rightarrow$ Spaces $_{*}$ is a diagram with the property that, for each $l \in \mathcal{L}$ there is a $k \in \mathcal{K}$ such that $\Theta(l) \sim \Omega \Phi(k)$.

If $X \in \widetilde{\mathcal{C}}_{\alpha}^{A}$, then $X$ is a retract of hocolim ${ }_{*} \Phi$, where $\Phi: \mathcal{K} \rightarrow \widetilde{\mathcal{C}}_{<\alpha}^{A}$, and so $\Omega X$ is a retract of $\operatorname{hocolim}_{*} \Theta$, where $\Theta(l) \sim \Omega \Phi(k)$ for some $\Phi(k) \in \widetilde{\mathcal{C}}_{<\alpha}^{\Omega A}$. Hence $\Theta(l) \in \widetilde{\mathcal{C}}_{<\alpha}^{\Omega A}$ by the inductive hypothesis, and $\Omega X \in \widetilde{\mathcal{C}}_{\alpha}^{\Omega A}$.

Here again we estimate the complexity of the spaces appearing in cellular inequalities due to Chachólski [C2].

Theorem 17. If $X$ is path connected, then the statements $\Sigma A \ll X$ and $A \ll \Omega X$ are equivalent, and

(a) $\kappa_{A}(\Omega X) \leq 2+\kappa_{\Sigma A}(X)$,

(b) $\kappa_{\Sigma A}(X) \leq \kappa_{A}(\Omega X)+1$.

Proof. When $A$ is not path connected, the result is trivial.

When $A$ is path connected, the equivalence of the two cellular inequalities is proved in [C2, Thm. 10.8]. First we consider the implication $\Sigma A \ll X \Rightarrow A \ll \Omega X$. From $\Sigma A \ll X$, we have the sequence of cellular inequalities

$$
A \ll \Omega \Sigma A \ll \Omega X .
$$

We now estimate the complexity by the computations

$$
\begin{aligned}
\kappa_{A}(\Omega X) & \leq \kappa_{A}(\Omega \Sigma A)+\kappa_{\Omega \Sigma A}(\Omega X) \\
& \leq 1+\left(1+\kappa_{\Sigma A}(X)\right) \\
& \leq 2+\kappa_{\Sigma A}(X)
\end{aligned}
$$


using Lemma [5] and Theorem 16(b). For the implication $A \ll \Omega X \Rightarrow \Sigma A \ll X$, we have the sequence of inequalities $\Sigma A \ll \Sigma \Omega X \ll X$, and the corresponding complexity estimate

$$
\kappa_{\Sigma A}(X) \leq \kappa_{\Sigma A}(\Sigma \Omega X)+\kappa_{\Sigma \Omega X}(X) \leq \kappa_{A}(\Omega X)+1
$$

using Lemma 5 and Proposition 7(a).

Remark. If we use $\widetilde{\kappa}_{A}$ instead of $\kappa_{A}$, the proof given above shows that $\mid \widetilde{\kappa}_{\Sigma A}(X)-$ $\widetilde{\kappa}_{A}(\Omega X) \mid \leq 1$.

We now turn to two more results of Chachólski, those concerning the delooping and desuspending of cellular inequalities. Note that, while the theorems of [C3] require only that $\mathcal{C}(A)=\mathcal{C}(\Sigma B)$ or that $\mathcal{C}(X)=\mathcal{C}(\Omega Y)$, our complexity estimate requires the stronger hypotheses $A \sim \Sigma B$ or $X \sim \Omega Y$.

Theorem 18. 1. If $A \sim \Sigma B$, then

(a) $\Omega A \ll \Omega X$ implies $A \ll X$, and

(b) $\kappa_{A}(X) \leq 1+\kappa_{\Omega A}(\Omega X)+1$.

2. If $X \sim \Omega Y$, then

(a) $\Sigma A \ll \Sigma X$ implies $A \ll X$, and

(b) $\kappa_{A}(X) \leq 2+\kappa_{\Sigma A}(\Sigma X)+1$.

Proof. The proof of the cellular inequality in (a) given in [C3, Thm. 9.7] goes as follows: we have

$$
B \ll \Omega \Sigma B \sim \Omega A \ll \Omega X, \quad \text { so } \quad A \sim \Sigma B \ll \Sigma \Omega X \ll X .
$$

It follows from the first sequence of inequalities, Lemma 5 and Theorem 14(a) that

$$
\kappa_{B}(\Omega X) \leq \kappa_{B}(\Omega \Sigma B)+\kappa_{\Omega \Sigma B}(\Omega X) \leq 1+\kappa_{\Omega A}(\Omega X) .
$$

We now compute

$$
\begin{aligned}
\kappa_{A}(X) & \leq \kappa_{\Sigma B}(\Sigma \Omega X)+\kappa_{\Sigma \Omega X}(X) \\
& \leq \kappa_{B}(\Omega X)+\kappa_{\Sigma \Omega X}(X) \\
& \leq 1+\kappa_{\Omega A}(\Omega X)+1
\end{aligned}
$$

using Lemma 5, Proposition 7(a) and Theorem 14(b). The proof of statement 2 is dual to the proof of statement 1 .

Corollary 19. If $A \sim \Omega B$ for some space $B$, then

$$
\max \left\{\kappa_{A}(X), \kappa_{A}(Y)\right\} \leq \kappa_{A}(X \times Y) \leq 4+\max \left\{\kappa_{A}(X), \kappa_{A}(Y)\right\} .
$$

Proof. As mentioned in the proof of Proposition 10(c), it suffices to estimate $\sup \left\{\kappa_{A}(X), \kappa_{A}(Y)\right\}$ for $\kappa_{A}(X)=\kappa_{A}(Y)=0$. If $X$ and $Y$ are retracts of wedges of copies of $A$, then $X \times Y$ is a retract of a product of such wedges; therefore it is enough to prove that if $X$ and $Y$ are wedges of copies of $A$, then $\kappa_{A}(X \times Y) \leq 4$. Using the splitting of a product after one suspension, we have

$$
\Sigma(X \times Y)=\Sigma A \wedge Z
$$

for some space $Z$, and so $\kappa_{\Sigma A}(\Sigma(X \times Y)) \leq 1$ by Proposition 8 (b). Since $A \sim \Omega B$, we may use Theorem [18(b) to desuspend the inequality and thereby obtain the estimate

$$
\kappa_{A}(X \times Y) \leq 2+\kappa_{\Sigma A}(\Sigma(X \times Y))+1 \leq 4 .
$$


2.4. Some examples. Before moving on to our study of good spaces, we apply our results to obtain some estimates of complexity concerning spheres and EilenbergMac Lane spaces.

Example 20. (1) For any path connected space $X, \Omega X \ll X$ and

$$
\kappa_{\Omega X}(X) \leq 2 .
$$

To see this, simply apply Lemma 5 , Proposition 7 and Theorem 14(b) to the sequence of inequalities $\Omega X \ll \Sigma \Omega X \ll X$.

(2) Since $K(G, n)=\Omega K(G, n+1)$ for any abelian group $G$, a simple induction using part (a) yields the estimate

$$
\kappa_{K(G, n)}(K(G, n+m)) \leq 2 m .
$$

(3) More generally, let $X_{0}, X_{1}, \ldots, X_{n}, \ldots$ be an infinite loop space, so $\Omega X_{n+1} \sim$ $X_{n}$. If we write $X_{\circ}$ to denote the basepoint component of $X$, then $X_{n}=$ $\Omega X_{n+1}=\Omega\left(\left(X_{n+1}\right)_{\circ}\right)$. Therefore $X_{n} \ll\left(X_{n+m}\right)_{\circ}$ and $\kappa_{X_{n}}\left(\left(X_{n+m}\right)_{\circ}\right) \leq$ $2 m$.

We finish this subsection with an application to complexity with respect to spheres. Stover has shown (using the Bousfield-Friedlander spectral sequence) that every connected space $X$ is a pointed homotopy colimit of a diagram of wedges of spheres [S]. In our language, $\kappa_{S^{1}}(X) \leq 1$; it was observed in [CDI, 9.6] that a simple modification of the argument shows that $\kappa_{S^{n}}(X) \leq 2$ for every space $X$ and every $n$.

Example 21. Here we show that the weaker bound $\kappa_{S^{n}}(X) \leq n+1$ can easily be proved by elementary methods (i.e., without spectral sequences). By definition, the $S^{n}$-complexity of $X$ is the same as the $S^{n}$-complexity of $C W_{S^{n}}(X)$, the $(n-1)$ connected cover of $X$. We will show that if $X$ is $(n-1)$-connected, then $\kappa_{S^{n}}(X) \leq$ $n+1$.

The inductive proof begins with Lemma 4. Let us assume then that $\kappa_{S^{n}}(X) \leq$ $n+1$ for every $(n-1)$-connected space $X$. If $Y$ is $n$-connected, then so is $\Sigma \Omega Y$, and

$$
\begin{aligned}
\kappa_{S^{n+1}}(Y) & \leq \kappa_{S^{n+1}}(\Sigma \Omega Y)+\kappa_{\Sigma \Omega Y}(Y) \\
& \leq \kappa_{S^{n}}(\Omega Y)+1 \\
& \leq n+2
\end{aligned}
$$

by Lemma 5. Proposition 7(a), Theorem 14(b), and the inductive hypothesis.

\section{Properties of good spaces}

We now turn our attention to the study of good spaces. This section has three main results: first we show that if $A$ is a good space, then so is $\Sigma A$; next we characterize good spaces; and finally we give an efficient construction of the functor $P_{A}$ under the assumption that $A$ is a good space.

3.1. Suspension of good spaces. The following fact will play a key role in the proof of our main theorem in $\S 4$.

Theorem 22. If $A$ is a good space, then $\Sigma A$ is also a good space. 
Proof. If $A$ is not path connected, then $\mathcal{C}(\Sigma A)=\mathcal{C}\left(S^{1}\right)$ is strongly closed, so we may assume that $A$ is path connected.

We have to show that $\mathcal{C}(\Sigma A)$ is closed under extensions by fibrations. So let $F \rightarrow E \rightarrow B$ be a fibration sequence with $\Sigma A \ll F$ and $\Sigma A \ll B$. Since $A$ is path connected, so are $F$ and $B$, and so $A \ll \Omega F$ and $A \ll \Omega B$ by Theorem 17, Looping the given fibration once yields the fibration sequence $\Omega F \rightarrow \Omega E \rightarrow \Omega B$. Since $A$ is a good space, $A \ll \Omega E$ and hence $\Sigma A \ll E$.

3.2. A characterization of good spaces. We show here that when $A$ is a good space, the fiber of the augmentation $X \rightarrow P_{A}(X)$ is naturally equivalent to $C W_{A}(X)$ in the homotopy category. In fact, this condition characterizes good spaces.

Theorem 23. For every space $A$ the following are equivalent:

(a) $\mathcal{C}(A)=\overline{\mathcal{C}(A)}$ (i.e., $A$ is a good space).

(b) For every space $X$, the sequence $C W_{A}(X) \stackrel{i}{\longrightarrow} X \stackrel{j}{\longrightarrow} P_{A}(X)$ is a fibration sequence.

(c) For each space $X \in \overline{\mathcal{C}(A)}$ there is a space $Y_{X}$ such that $\left[A, Y_{X}\right]=*$ and $C W_{A}(X) \rightarrow X \rightarrow Y_{X}$ is a fibration sequence.

Proof. To prove that (a) implies (b), we begin with the fibration sequence

$$
\bar{P}_{A}(X) \stackrel{l}{\longrightarrow} X \stackrel{j}{\longrightarrow} P_{A}(X)
$$

and show that there is a weak equivalence $\theta: C W_{A}(X) \rightarrow \bar{P}_{A}(X)$ such that the diagram

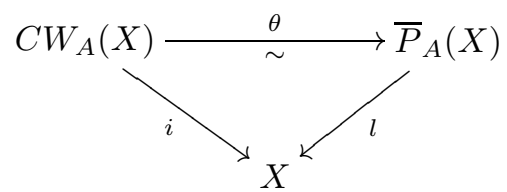

commutes up to homotopy. Since $i: C W_{A}(X) \rightarrow X$ is characterized up to weak equivalence by the properties (1) $C W_{A}(X)$ is $A$-cellular, and (2) $i$ is a $A$ equivalence $\mathrm{Fa} 2$, it is enough to verify that these properties are satisfied by the map $l: \bar{P}_{A}(X) \rightarrow X$. Property (1) follows from $\bar{P}_{A}(X) \in \overline{\mathcal{C}(A)}=\mathcal{C}(A)$ C2, Thm. 17.1]. For (2), use the fibration sequence $\bar{P}_{A}(X) \stackrel{l}{\longrightarrow} X \stackrel{j}{\longrightarrow} P_{A}(X)$ to obtain the fibration sequence

$$
\operatorname{map}_{*}\left(A, \bar{P}_{A}(X)\right) \stackrel{l_{*}}{\longrightarrow} \operatorname{map}_{*}(A, X) \stackrel{j_{*}}{\longrightarrow} \operatorname{map}_{*}\left(A, P_{A}(X)\right) .
$$

The map $l_{*}$ is a weak equivalence because $\operatorname{map}_{*}\left(A, P_{A}(X)\right) \sim *$ by definition [Fa2, 1.A.4].

Clearly (b) implies (c): simply take $Y_{X}=P_{A}(X)$.

Now assume that (c) holds. By [CPS, Thm.1.5], there is a good space $G$ such that $\mathcal{C}(A) \subseteq \overline{\mathcal{C}(A)}=\mathcal{C}(G)$. It remains to prove the reverse containment. Let $X \in \mathcal{C}(G)$; we will show that $X \in \mathcal{C}(A)$. The basepoint component of the space $\operatorname{map}_{*}\left(A, Y_{X}\right)$ is contractible because $C W_{A}(X) \rightarrow X$ is an $A$-equivalence. Since $\left[A, Y_{X}\right]=*, \operatorname{map}_{*}\left(A, Y_{X}\right)$ is path connected, so $Y_{X}$ is $A$-null. Thus we have the 
commutative diagram

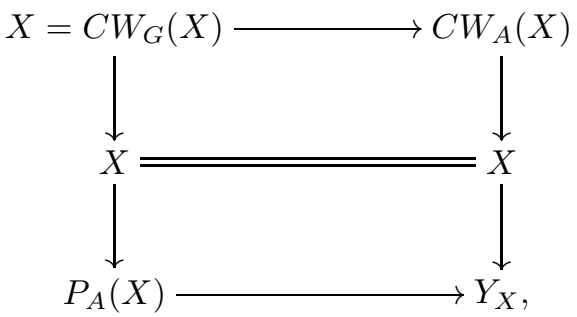

which shows that $X$ is a homotopy retract of $C W_{A}(X) \in \mathcal{C}(A)$. Since $\mathcal{C}(A)$ is closed under retracts [Fa2, 2.E.11], this implies that $X \in \mathcal{C}(A)$, verifying (a).

Remark. The argument given above can be generalized: if $\overline{\mathcal{C}(A)}=\mathcal{C}(B)$, then there is a weak equivalence $\theta: C W_{B} \rightarrow \bar{P}_{A}$ which is natural in the homotopy category. It is not clear whether or not $\theta$ can be defined in a natural way on the point-set level.

3.3. An easy construction of $P_{A}(X)$. In this section we derive an efficient construction of $P_{A}(X)$ under the assumption that $A$ is a good space. This construction plays a key role in the proof of the main theorem in Section 4 .

We set $X^{(0)}=X$ and inductively define $X^{(k+1)}$ as the cofiber of the evaluation map

$$
\bigvee_{f \in\left[\Sigma^{k} A, X^{(k)}\right]}\left(\Sigma^{k} A\right)_{f} \stackrel{\epsilon}{\longrightarrow} X^{(k)}
$$

given by $\left.\epsilon\right|_{\left(\Sigma^{k} A\right)_{f}}=f$. Starting with the inclusion $i_{1}: X \hookrightarrow X^{(1)}$, we let $i_{k}: X \rightarrow$ $X^{(k)}$ be the unique map making the diagram

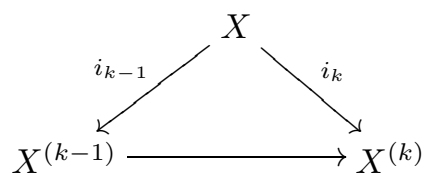

commutative. Let $X^{(\infty)}$ be the telescope of the sequence $\cdots \rightarrow X^{(k)} \rightarrow X^{(k+1)} \rightarrow$ $\cdots$ and write $i: X \rightarrow X^{(\infty)}$ for the map induced by the maps $i_{k}$.

Theorem 24. For connected $C W$ complexes $A$ and $X$, there is a homotopy equivalence $\phi: X^{(\infty)} \rightarrow P_{A}(X)$ making the diagram

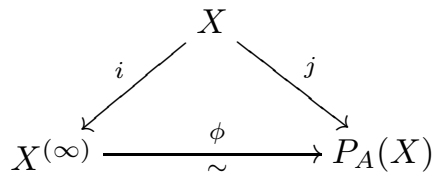

homotopy commutative.

Proof. We claim that the composite map

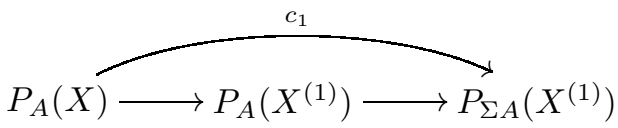


is a weak equivalence. Consider the commutative diagram

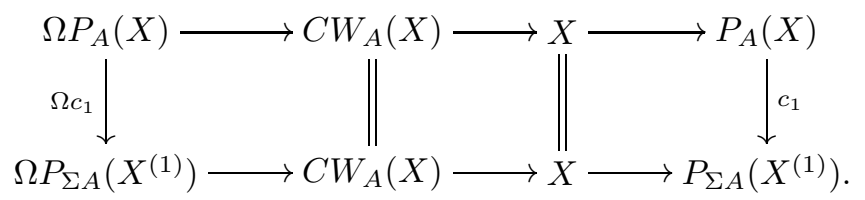

Here the top row is a fibration sequence by Theorem 23 since $A$ is a good space, and the bottom row is a fibration sequence by [C2, Thm. 20.3]. From the diagram we see that $\Omega c_{1}: \Omega P_{A}(X) \rightarrow \Omega P_{\Sigma A}\left(X^{(1)}\right)$ is a weak equivalence. Since $A$ is path connected, so are $P_{A}(X)$ and $P_{\Sigma A}\left(X^{(1)}\right)$. This implies that $c_{1}: P_{A}(X) \rightarrow$ $P_{\Sigma A}\left(X^{(1)}\right)$ is a weak equivalence.

Now a simple induction using Theorem 22 shows that each of the natural maps $P_{\Sigma^{k} A}\left(X^{(k)}\right) \rightarrow P_{\Sigma^{k+1} A}\left(X^{(k+1)}\right)$ is a weak equivalence. These maps together constitute a telescope diagram $\Phi: \omega \rightarrow$ Spaces $_{*}$. On taking the homotopy colimit, we obtain a weak equivalence

$$
c: P_{A}(X) \longrightarrow \text { hocolim }_{*} \Phi .
$$

By construction of the functor $P_{\Sigma^{k} A}$, the map $l_{k}: X^{(k)} \rightarrow P_{\Sigma^{k} A} X^{(k)}$ is a $(k+1)$ equivalence. It follows that the limit map

$$
l: X^{(\infty)} \longrightarrow \operatorname{hocolim}_{*} \Phi
$$

is a weak equivalence. Since all spaces are $\mathrm{CW}$ complexes, $c$ is invertible, and the map $\phi=c^{-1} \circ l$ is the desired homotopy equivalence.

Remark. Even without the hypothesis that $A$ and $X$ are CW complexes, we can conclude that $P_{A}(X)$ and $X^{(\infty)}$ are weakly equivalent.

\section{Complexity and good spaces}

In this final section we bring together much of our previous work in order to prove an upper bound for $\kappa_{\Sigma A}(X)$ when $A$ is a good space. This upper bound is independent of $A$ and $X$.

Theorem 25. If $A$ is a good space, then

$$
\kappa_{\Sigma A}(X) \leq \omega+1 \quad \text { for every space } X .
$$

Proof. If $A$ is not path connected, then $S^{1}$ is a retract of $\Sigma A$, so $\kappa_{A}(X) \leq \kappa_{S^{1}}(X) \leq$ 1 for all $X$. Thus we restrict our attention to path connected spaces $A$. It follows, of course that all spaces that are $A$-cellular, or $\Sigma A$-cellular, are also path connected.

We will use the notation of $\S 3.3$. Assume first that $X \sim \Sigma Y$ and that $A \ll Y$. Then $Y^{(\infty)} \sim P_{A}(Y) \sim *$. Now consider the diagram

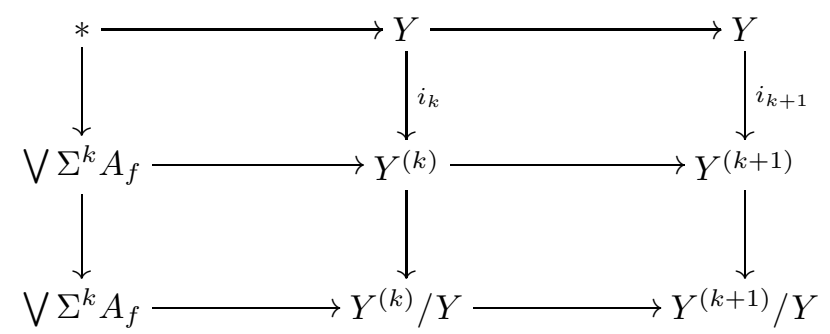


whose rows and columns are cofiber sequences. In particular, the quotient $Y^{(k+1)} / Y$ is the homotopy pushout of the diagram

$$
* \longleftarrow \Sigma^{k} A_{f} \longrightarrow Y^{(k)} / Y
$$

for $k \geq 0$. Clearly $Y=Y^{(0)}$ implies $Y^{(0)} / Y \sim *$, and hence $Y^{(1)} / Y \sim \Sigma\left(\bigvee A_{f}\right) \in$ $\mathcal{C}_{0}^{\Sigma A}$. We conclude by induction that $Y^{(k)} / Y \in \mathcal{C}_{k}^{\Sigma A}$ for each $0<k<\omega$ and hence that $X \sim \Sigma Y \sim Y^{(\infty)} / Y \in \mathcal{C}_{\omega}^{\Sigma A}$.

Now we handle the general case. The complexity of $X$ is defined to be $\kappa_{\Sigma A}(X)=$ $\kappa_{\Sigma A}\left(C W_{\Sigma A}(X)\right)$, so we replace $X$ with $C W_{\Sigma A}(X)$; that is, we assume that $\Sigma A \ll X$ to begin with. Then we have

$$
\Sigma A \ll X \text { implies } A \ll \Omega X .
$$

Therefore $\Sigma A \ll \Sigma \Omega X$ and $\kappa_{\Sigma A}(\Sigma \Omega X) \leq \omega$ by the special case discussed in the previous paragraph. Now we apply Lemma 5 and Theorem 14(b) to conclude that

$$
\kappa_{\Sigma A}(X) \leq \kappa_{\Sigma A}(\Sigma \Omega X)+\kappa_{\Sigma \Omega X}(X) \leq \omega+1,
$$

which completes the proof.

Corollary 26. If $A$ is a good space and $A \ll \Omega Y$, then $\kappa_{A}(\Omega Y) \leq \omega+1$.

Proof. If $A$ is not path connected, the result is trivial. If $A$ is path connected, then so is $\Omega Y$ because $A \ll \Omega Y$. Then we have $\Sigma A \ll Y$, and by Theorem 25 we have $\kappa_{\Sigma A}(Y) \leq \omega+1$. Now we apply Theorem 17 to obtain

$$
\kappa_{A}(\Omega Y) \leq 2+\kappa_{\Sigma A}(Y) \leq 2+\omega+1=\omega+1,
$$

using ordinal arithmetic for the final step.

\section{ACKNOWLEDGEMENT}

We are grateful to Wojciech Chachólski for helpful advice at many stages in the development and preparation of this paper.

\section{REFERENCES}

[A] M. Arkowitz, The generalized Whitehead product, Pacific J. Math. 12 (1962), 7-23. MR0155328 (27:5262)

[B-K] A. K. Bousfield and D. M. Kan, Homotopy limits, completions and localizations, SLNM 304 Springer-Verlag, Berlin, 1972. MR0365573(51:1825)

[B] A. K. Bousfield, Localization and periodicity in unstable homotopy theory, J. Am. Math. Soc. 7 (1994), 831-874. MR.1257059 (95c:55010)

[C1] W. Chachólski, Closed classes, Algebraic topology: new trends in localization and periodicity (Sant Feliu de Guíxols, 1994), 95-118, Progr. Math., 136, Birkhäuser, Basel, 1996. MR.1397724 (97e:55007)

[C2] W. Chachólski, On the functors $C W_{A}$ and $P_{A}$, Duke Math. J. 84 (1996), 599-631. MR.1408539 (97i:55023)

[C3] W. Chachólski, Desuspending and delooping cellular inequalities, Invent. Math. 129 (1997), 37-62. MR.1464865 (98i:55013)

[CDI] W. Chachólski, W. G. Dwyer and M. Intermont, The A-complexity of a space, J. London Math. Soc. (2) 65 (2002), 204-222. MR1875145 (2002j:55009)

[CPS] W. Chachólski, P.-E. Parent and D. Stanley, Cellular generators, Proc. Amer. Math. Soc. 132 (2004), no. 11, 3397-3409. MR2073317 (2005h:55012)

[Fa1] E. Dror Farjoun, Cellular Inequalities, The Cech Centennial (Boston, MA, 1993), 159-181, Contemp. Math., 181, AMS, Providence, RI, 1995. MR1320991 (96g:55011)

[Fa2] E. Dror Farjoun, Cellular Sapces, Null Spaces, and Homotopy Localization, SpringerVerlag, Berlin, 1996. 
[Fr] A. A. Fraenkel, Abstract Set Theory, North-Holland Publishing Co., Amsterdam, 1966. MR0197271 (33:5442)

[S] C. R. Stover, A van Kampen spectral sequence for higher homotopy groups, Topology 29 (1990), 9-26. MR.1046622 (91h:55011)

[W] G. W. Whitehead, Elements of Homotopy Theory, Graduate Texts in Mathematics 61, Springer-Verlag, 1978. MR0516508 (80b:55001)

Department of Mathematics, Kalamazoo College, Kalamazoo, Michigan 49006

E-mail address: intermon@kzoo.edu

Department of Mathematics, Western Michigan University, Kalamazoo, Michigan 49008

E-mail address: Jeff.Strom@wmich.edu 